\title{
Biologically active and reserve substances of Siberian peonies
}

\author{
Vera A. Kostikova ${ }^{1 *}$, Olga V. Kalendar ${ }^{1}$, Nikolai A. Tashev ${ }^{2}$, Andrey S. Erst ${ }^{1}$, and Olga Yu. \\ Vasilyeva $^{1}$ \\ ${ }^{1}$ Central Siberian Botanical Garden, Siberian Branch of Russian Academy of Sciences (CSBG SB \\ RAS), Zolotodolinskaya Str., 101, Novosibirsk 630090, Russia \\ ${ }^{2}$ University of Forestry, Faculty of Business Management (FBM), Sofia 1756, Bulgaria
}

\begin{abstract}
Levels of biologically active and reserve substances were investigated in the leaves and rhizomes of Paeonia anomala L., P. hybrida Pall., and P. lactiflora Pall. The peonies' leaves are rich in ascorbic acid $(1205.7 \mathrm{mg} / 100 \mathrm{~g}$ in $P$. anomala) and tannins (15.9\% in P. lactiflora and $15.7 \%$ in $P$. anomala) at the beginning of the growth season. Concentrations of flavonols, pectins, and carotenoids in the Siberian peonies are low at the beginning of the growth season. P. lactiflora is a promising species for further research on the set and levels of biologically active and reserve substances in rhizomes because this species stands out among the three Siberian species in terms of the levels of catechins (1297.8 $\mathrm{mg} / 100 \mathrm{~g})$, protopectins $(10.1 \%)$, saponins $(19.2 \%)$, and sugars $(22.1 \%)$ in rhizomes. In these parts of the plant, starch (18.6\%) accumulates the most in $P$. hybrida among the studied peonies species. Concentrations of the analyzed substances in the rhizomes of the peonies change significantly by the end of the growth season and vary widely among these species. Levels of ascorbic acid (except for $P$. hybrida), protopectins, and starch increase significantly while concentrations of catechins and sugars decrease in the rhizomes by the end of the growth season.
\end{abstract}

\section{Introduction}

The genus Paeonia L. includes 33 species, distributed mainly in Europe, the Mediterranean, and East and Southeast Asia [1]. There are three species in the flora of Siberia: P. anomala L., P. hybrida Pall., and P. lactiflora Pall. [2].

The Siberian species belong to two sections: Flavonia Kem.-Nath. (P. lactiflora) and Sternia Kem.-Nath. (P. anomala and P. hybrida). P. lactiflora has one to seven milky-white flowers per shoot, while $P$. anomala and $P$. hybrida have one purple-pink flower per shoot [1]. The leaves of $P$. lactiflora are double trifoliate, in contrast to the triple trifoliate pinnate leaves of $P$. anomala and $P$. hybrida [3]. Paeonia hybrida reproduces via numerous spindle-shaped tuberous parts of lateral roots, unlike the other two species, which usually reproduce as part of the rhizome with a bud at the base of the shoot [4].

\footnotetext{
*Corresponding author: serebryakova-va@yandex.ru
} 
According to studies, early flowering P. lactiflora, P. anomala, and P. hybrida are of great interest in the harsh climate of Siberia. In addition, the forest species P. lactiflora and $P$. anomala have the longest growth season and accordingly are characterized by the preservation of decorative features.

Wild species of peonies and the varieties created from them are ornamental plants and have medicinal properties. Peonies contain a wide range of biologically active substances belonging to various classes of chemical compounds: carbohydrates, terpenoids, steroids, phenol carboxylic acids, phenolic glycosides, flavonoids, tannins, vitamins, iridoids, and trace elements [5-7]. Only $P$. anomala $\mathrm{L}$. is used for medicinal purposes in conventional medicine [8]. Nonetheless, the amount of this species collected in the wild as raw materials for industrial purposes is currently limited due to the small size of its wild populations. Therefore, it is important to investigate the chemical composition and medicinal effects of other species that either grow in this region or have a high potential for introduction into an environment with these climatic conditions.

The aim of this study was to quantify biologically active and reserve substances in the leaves and rhizomes of the P. lactiflora, $P$. anomala, and P. hybrida introduced into the forest-steppe environment of the Novosibirsk Region (Oblast).

\section{Materials and methods}

The leaves and rhizomes of P. lactiflora, $P$. anomala, and $P$. hybrida were collected at the beginning of the growth season (in May 2017) and at its end (17 August 2017) from "Collections of living plants indoors and outdoors" (unique scientific unit No. 440534 of CSBG SB RAS) in a garden plot located at a forest-steppe site with gray forest soil and a mean yearly temperature/precipitation of $1.8{ }^{\circ} \mathrm{C} / 448 \mathrm{~mm}$. The raw materials were dried and crushed to obtain a representative sample for the analysis.

The quantitation of flavonols was carried out by the spectrophotometric aluminum chloride method. Concentrations of flavonols were calculated as rutin equivalents (SigmaAldrich) [9]. The level of catechins was measured by a technique based on the ability of catechins to give crimson staining when mixed with a solution of vanillin in concentrated hydrochloric acid. The conversion factor was calculated using catechin from a commercial source (Sigma-Aldrich) [10]. The quantitation of tannins (hydrolyzable tannins) was performed via the approach proposed by L.M. Fedoseeva [11], where tannin from SigmaAldrich served as a standard sample. The quantification of ascorbic acid was carried out by titration. This technique is based on the reducing properties of ascorbic acid [12]. Pectin substances (pectins and protopectins) were quantitated by a carbazole-free method based on specific yellow-orange staining of uronic acids in the presence of thymol in a sulfuric-acid solution. The concentrations of pectin substances were calculated as galacturonic-acid equivalents [13]. Levels of saponins were determined by the gravimetric method [14]. Levels of carotenoids was determined in an acetone-ethanol extract [12, 15]. Sugars were quantified by the method of A.S. Shvetsov and E.H. Lukyanenko, which is based on potassium ferricyanide reduction by reducing sugars to ferricyanide in an alkaline solution. The sugars were quantitated using a calibration curve plotted by means of glucose standards [12]. To quantify starch, the raw material was hydrolyzed with a hydrochloric acid solution $(10 \mathrm{~g} / \mathrm{l})$ for $6 \mathrm{~h}$. Because glucose constitutes a 0.89996 proportion of starch by weight, the resulting difference (in \%) was multiplied by this quotient, and starch content of the analyzed samples was determined in this way [16]. All the experiments were set up with two biological replicates and three technical replicates per treatment. All biochemical parameters were calculated for the mass of absolutely dry raw materials. 


\section{Results and discussion}

First, the concentrations of phenolic compounds-flavonols, catechins, and tannins-and those of ascorbic acid, pectin substances, saponins, and carotenoids were determined in the leaves and rhizomes of the three Paeonia species at the beginning of the growth season (Table 1). It was revealed that flavonol levels in the leaves are almost identical between the peonies of the section Sternia ( $P$. anomala and $P$. hybrida); these concentrations are slightly higher than those in the leaves of $P$. lactiflora. It should be noted that the levels of flavonols in the peonies' leaves were rather low at the beginning of the growth season. Flavonols were not detectable in the rhizomes of the studied peonies. The concentration of tannins was found to be higher in the leaves of $P$. lactiflora and $P$. anomala than in their rhizomes (two- to threefold). On the contrary, levels of catechins are higher in the underground organs of $P$. lactiflora and $P$. anomala than in their leaves, whereas in $P$. hybrida, these levels are higher in the leaves. P. lactiflora stands out among the three analyzed species in terms of the catechin content of the rhizomes $(1297.8 \mathrm{mg} / 100 \mathrm{~g})$. The concentration of ascorbic acid turned out to be six- or elevenfold higher in the leaves of the peonies than in their rhizomes. The highest level of ascorbic acid was registered in the leaves of $P$. anomala $(1205.7 \mathrm{mg} / 100 \mathrm{~g})$. The concentration of pectins in the leaves and rhizomes of the peonies is rather low, and similarly to catechins, the concentration of pectins in $P$. lactiflora and $P$. anomala is higher in the rhizomes than leaves, whereas in $P$. hybrida, it is higher in the leaves. The level of protopectins was found to significantly exceed that of pectins. In rhizomes, the concentration of protopectins is the highest in $P$. lactiflora $(10.1 \%)$, while in the two species of the Sternia section, this concentration is within $6-7 \%$. The concentration of protopectins proved to be higher in the underground organs of the peonies than in their leaves. The level of saponins is almost identical between the rhizomes and leaves of the three peonies. Their highest concentration was found in the leaves and rhizomes of $P$. lactiflora (19\%). Carotenoids, just as flavonols, are present only in the peonies' leaves. Their concentration is the highest in the leaves of the section Sternia species ( $P$. anomala and $P$. hybrida): $77.7 \mathrm{mg} / 100 \mathrm{~g}$ and $56.9 \mathrm{mg} / 100 \mathrm{~g}$, respectively, which is still rather low.

Next, reserve substances (starch and sugars) in the leaves and rhizomes of the Siberian peonies were quantified at the beginning of the growth season (Table 1). It was revealed that the peonies of the Sternia section contain more starch in the rhizomes as compared to $P$. lactiflora. P. hybrida is remarkable in this regard: the rhizome starch level reaches $18.6 \%$. The sugar concentration is the highest in P. lactiflora rhizomes and $P$. hybrida leaves. It must be mentioned that in P. lactiflora, this concentration is higher in the rhizomes than in the leaves, whereas in the other two species, the opposite is true.

In addition, the levels of biologically active and reserve substances in the rhizomes of the Siberian peonies were determined at the end of the growth season. The levels of protopectins in the rhizomes of all three peonies $(11.3 \%$ in P. lactiflora, $15.9 \%$ in $P$. anomala, and $8.16 \%$ in $P$. hybrida) are higher at the end of the growth season than at its beginning (Fig. 1). On the contrary, the concentration of catechins $(245.3 \mathrm{mg} / 100 \mathrm{~g}$ in $P$. lactiflora, $60.3 \mathrm{mg} / 100 \mathrm{~g}$ in $P$. anomala, and $45.8 \mathrm{mg} / 100 \mathrm{~g}$ in $P$. hybrida) decreases toward the end of the growth season (Fig. 2). The level of ascorbic acid in the rhizomes of $P$. lactiflora $(113.6 \mathrm{mg} / 100 \mathrm{~g})$ and $P$. anomala $(158.7 \mathrm{mg} / 100 \mathrm{~g})$ is higher at the end of the growth season, whereas in $P$. hybrida $(85.9 \mathrm{mg} / 100 \mathrm{~g})$, it is higher at the beginning (Fig. 2). The concentration of pectins in the rhizomes of $P$. anomala $(0.9 \%)$ and $P$. hybrida $(1.0 \%)$ is higher at the end of the growth season, whereas in P. lactiflora $(0.4 \mathrm{mg} / 100 \mathrm{~g})$, it is higher at the beginning. Levels of saponins in rhizomes barely changed in P. lactiflora $(18.9 \%)$ and P. anomala (12.2\%) during the growth season but doubled in P. hybrida (12.3\%; Fig. 1). The sugar level in rhizomes significantly decreased (to $7.6 \%$ in P. lactiflora, to $4.8 \%$ in $P$. 
anomala, and to $5.0 \%$ in $P$. hybrida), while the starch level doubled (to $21.8 \%$ in $P$. lactiflora, to $31.2 \%$ in $P$. anomala, and to $28.6 \%$ in $P$. hybrida) as compared to the beginning of the growth season (Fig. 1).

Table 1. Concentrations of biologically active and reserve substances in Siberian peonies at the beginning of the growth season

\begin{tabular}{|l|c|c|c|c|c|c|}
\hline \multirow{2}{*}{ Substances } & \multicolumn{2}{|c|}{$\begin{array}{c}\text { P. lactiflora } \\
22.05 .2017\end{array}$} & \multicolumn{2}{c|}{$\begin{array}{c}\text { P. anomala } \\
22.05 .2017\end{array}$} & \multicolumn{2}{c|}{$\begin{array}{c}\text { P. hybrida } \\
\text { 25.05.2017 }\end{array}$} \\
\cline { 2 - 7 } & leaves & rhizomes & leaves & rhizomes & leaves & rhizomes \\
\hline Moisture, \% & 85.70 & 78.60 & 82.06 & 77.61 & 79.56 & 84.39 \\
\hline Flavonols, \% & 1.47 & no & 1.95 & no & 1.96 & no \\
\hline Catechins, mg/100g & 103.50 & 1297.80 & 76.40 & 81.30 & 96.40 & 76.90 \\
\hline Tannins, \% & 15.87 & 5.47 & 15.72 & 6.48 & 12.57 & 6.13 \\
\hline Ascorbic acid, mg/100g & 845.45 & 90.65 & 1205.61 & 87.54 & 868.40 & 203.08 \\
\hline Pectins, \% & 0.41 & 0.74 & 0.68 & 0.74 & 0.63 & 0.53 \\
\hline Protopectins, \% & 4.13 & 10.10 & 5.96 & 6.91 & 4.45 & 5.68 \\
\hline Saponins, \% & 19.21 & 18.94 & 12.06 & 12.69 & 6.47 & 7.23 \\
\hline Carotenoids, mg/100g & 19.70 & no & 77.70 & no & 56.90 & no \\
\hline Sugar, \% & 18.95 & 22.06 & 17.61 & 13.62 & 20.65 & 12.36 \\
\hline Starch, \% & - & 12.19 & - & 15.80 & - & 18.64 \\
\hline
\end{tabular}

Note: no, not found; “-“", not tested.

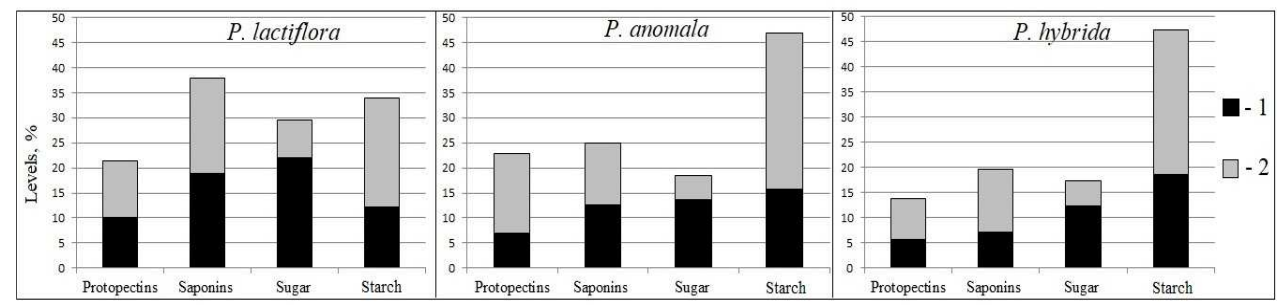

Fig. 1. Levels of protopectins, saponins, sugars, and starch in the rhizomes of the Siberian peonies at the beginning (1) and at the end (2) of the growth season.
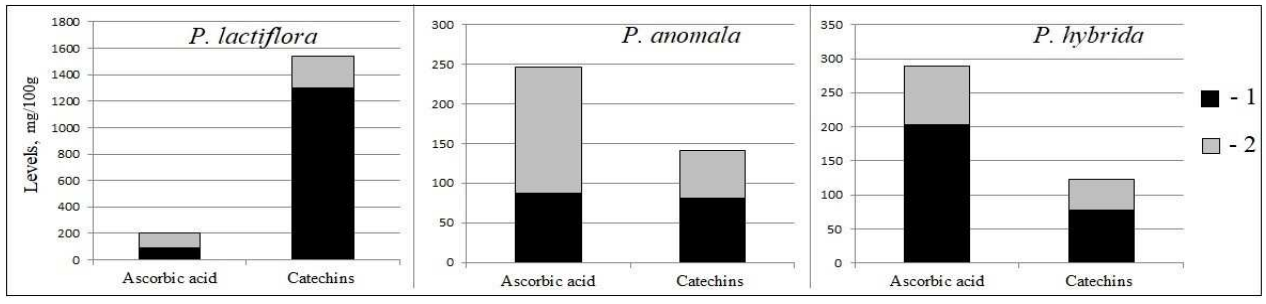

Fig. 2. Levels of ascorbic acid and catechins in the rhizomes of the three peonies at the beginning (1) and at the end (2) of the growth season. 


\section{Conclusion}

The leaves and rhizomes of the Siberian species of peonies grown on the territory of the CSBG SB RAS (Novosibirsk) contain biologically active and reserve substances in sufficient amounts for their further study as additional sources of medicinal raw materials for the pharmaceutical industry and for rational use of Siberian phytoresources.

The work was supported by a publicly funded project of the CSBG SB RAS, No. AAAA-A21-121011290025-2.

Acknowledgements. The English language was corrected and certified by shevchukediting.com

\section{References}

1. D. Y. Hong, Peonies of the world. Pt. 1: Taxonomy and phytogeography (London, 2010)

2. N. V. Friesen, Flora of Siberia, 6 (1993)

3. O. V. Komina, Biological characteristics of some species of the genus Paeonia L. introduced into the forest-steppe zone of Western Siberia (Novosibirsk, 2014)

4. I. V. Vereshchagin, Wild Peonies of Altai (Barnaul, 2003)

5. B. N. Golovkin, I. A. Rudenskaya, I. A. Trofimova et al., Biologically active substances of vegetable origin (Moscow, 2001)

6. F. Zhang, J. Qu, K. Thakur et al., Food chemistry, 285 (2019)

7. N. N. Tong, X. Y. Zhou, L. P. Peng et al., Journal of Ethnopharmacology, 273 (2021)

8. Pharmacopeial article 42-531-98. Rhizomata et radices Paeonia anomala (Moscow, 2000)

9. V. V. Belikov, Farmatsiya, 1 (1970)

10. T .A. Kukushkina, A. A. Zykov, L. A. Obukhova, Manzhetka obyknovennaya (Alchemila vulgaris) kak istochnik lekarstvennyh sredstv, in Proceedings of the VII International Congress on Actual problems of creating new drugs of natural origin, 3-5 Yuly 2003, St. Petersburg (2003)

11. L. M. Fedoseyeva, Plant chemistry, 2 (2005)

12. A. I. Ermakov, Methods of biochemical study of plants (Leningrad, 1987)

13. V. I. Kriventsov, Works of the Nikitsky Botanical Garden, 109 (1989)

14. A. V. Kiseleva, T. A. Volkhonskaya, V. Ye. Kiselev, Biologically active substances of medicinal plants in Southern Siberia (Novosibirsk, 1991)

15. V. I. Kriventsov, Methodological recommendations for the analysis of fruits for biochemical composition (Yalta, 1982)

16. V. Ya. Borodova, E. S. Gorenkov, O. A. Klyueva et al., Methodical instructions for the chemical-technological variety testing of vegetable, fruit and berry crops for the canning industry (Moscow, 1993) 